\title{
In Response: Study Not Designed To Address Socio-Economic Issues
}

We are pleased to address the questions that you raised and expand the dialogue regarding a novel approach to chronic pain management described in the "Feasibility of a rapid opioid rotation and titration" study. This was an exploratory pilot study to investigate the feasibility and safety of quickly converting chronic pain patients from one opioid to another. As described in the paper, the 24-hour inpatient stay for intravenous
(IV) patient controlled analgesia (PCA) was a safety requirement of the Mount Sinai IRB. Rapid opioid rotation and titration (RORT) was well tolerated with good efficacy and patient satisfaction. A shorter protocol (< 8 hours IV-PCA) could allow RORT to be an outpatient procedure and addresses the burdens of an inpatient stay. The results of our current study provide the foundation for such future research. 
You aptly pointed out the trend in the United States for pain specialists, despite their scarcity, to manage chronic pain patients for many primary care physicians due to opioids' complexity. While we agree that handling issues of rotation and titration is common for a pain specialist, a procedure such as RORT could standardize and shorten the process. RORT could allow primary care physicians who are less comfortable with opioids to defer the interventional aspect of rotation and titration to pain specialists. As discussed in our paper, pain specialists could assume responsibility for the interventional and expert management of opioid switching without the need for protracted patient care. Patients could be referred back to their primary care physician with a specific treatment plan. Our research is appropriate for a variety of physicians with greater exposure to and expertise in chronic pain management, including physiatrists, neurologists, palliative care specialists, and oncologists.

The letter also raises doubts about the practicality of using an IV form of oxymorphone. Importantly, our findings could be generalized to other opioids that have both an IV and oral formulation. We used the IV oxymorphone in our study to keep the opioid consistent with the oral oxymorphone (OPANA ER and IR) provided by our sponsor ENDO Pharmaceuticals.
Our study is not designed to address the socio-economic complexities of the health insurance payment system in the United States. Chronic pain management is a growing problem all over the world, and our novel approach might be utilized in other countries with a different health insurance system. It is our hope that this novel approach might encourage appropriate medical specialists to adapt existing billing codes or push for new ones to provide more effective pain treatment for the rapidly expanding chronic pain patient population. Further research into a shorter outpatient RORT proto$\mathrm{col}$ ( $<8$ hours IV-PCA) is needed. Sincerely,

Marina Korkmazsky, MS, OMS-III

Clinical Research Professor

The Mount Sinai

Medical Center,

Voorhees, NJ

E-mail: mkork15@gmail.com

Marco Pappagallo, MD

Professor of Anesthesiology

Department of Anesthesiology

The Mount Sinai Medical Center

New York, NY 10029 USA

Email: marco.pappagallo@mssm.edu 